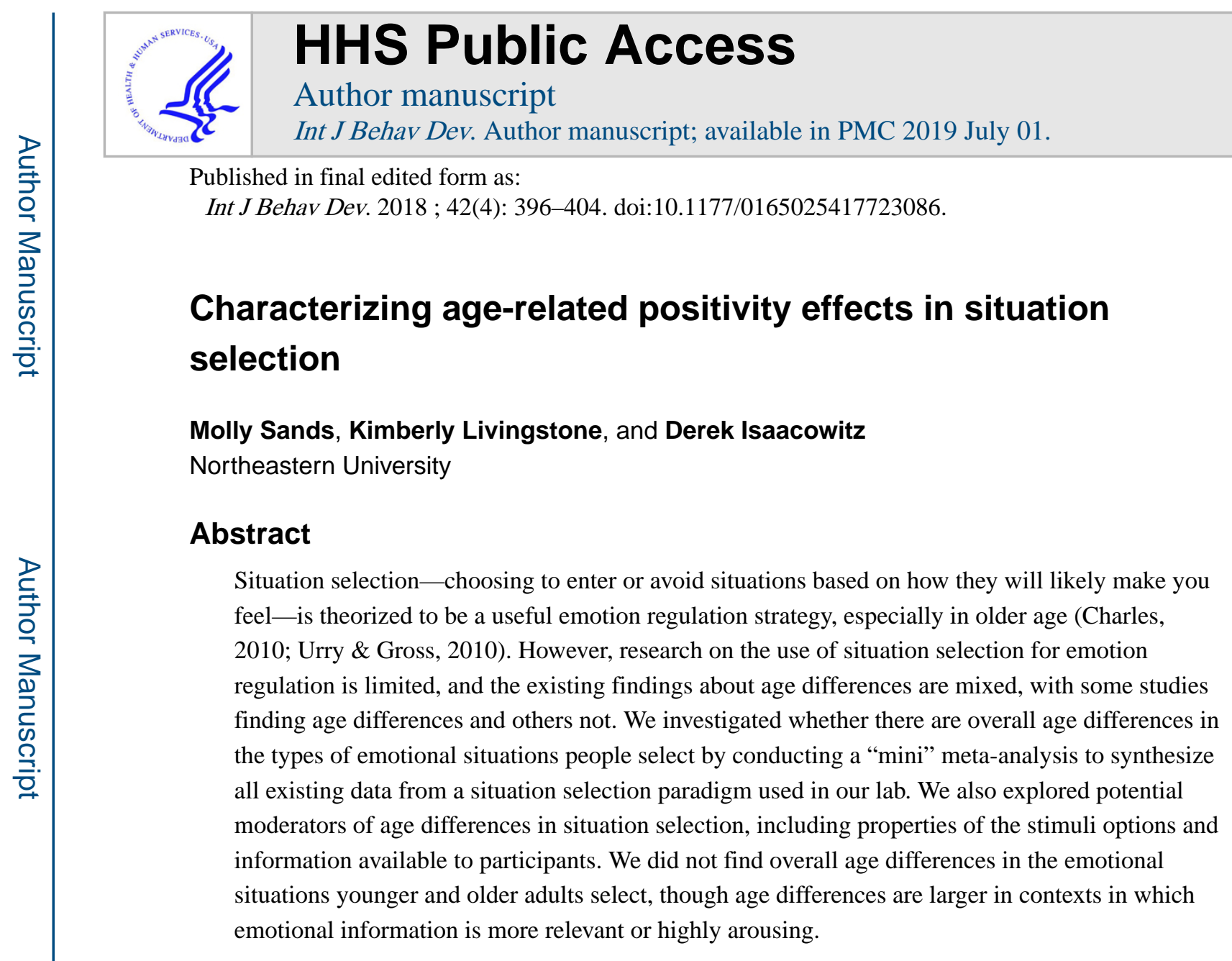

\title{
Keywords
}

aging; emotion; affect; situation selection; positivity effect

\begin{abstract}
Every day people make countless choices that may influence their emotional lives. Do we stay late to finish a project at work or join our co-workers for dinner? Do we feel frustrated while sitting in rush hour traffic or leave for work thirty minutes earlier? Do we watch a light-hearted comedy or a documentary about the spread of malaria? These types of decisions about emotional situations and the emotional content we choose to engage with allow us to construct the emotional nature of our lives, and are therefore one way we can manage our emotions.
\end{abstract}

Choices to enter or avoid situations based on how they will likely make one feel—known as situation selection-may be a particularly important means of emotion regulation, especially in later life. The ability to effectively use situation selection to manage one's emotions is theorized to rely primarily on resources that increase with age, such as emotional knowledge and affective forecasting (e.g., Scheibe, Mata \& Carstensen, 2011), and to be less dependent on cognitive and physiological processes that decline with age (Charles, 2010). Despite strong theoretical reasoning about situation selection becoming more important with age

Please address all correspondence to: Derek M. Isaacowitz, Department of Psychology, Northeastern University, 360 Huntington Ave., Boston, MA 02115,dmi@neu.edu. 
(Charles, 2010; Urry \& Gross, 2010), research on the use of situation selection for emotion regulation is limited, and the existing findings on age differences (primarily from our lab) are mixed.

Below, we review the theoretical case for the importance of situation selection for emotion regulation in older age and summarize existing, mixed research on the topic. We then present a "mini meta-analysis" that synthesizes the results of all the situation selection studies we have conducted in our lab. Since most of the research on age differences in situation selection has used this paradigm, a mini meta-analysis allows us to assess the current empirical support for the idea that older adults use positivity in selections to maximize emotional well-being.

\section{Age Differences in Emotion Regulation}

Emotion regulation is the process by which people can increase, decrease, or maintain positive and negative feelings. There are many ways individuals can regulate-or managetheir emotions (Gross \& Thompson, 2007). Of particular importance in the context of aging is the distinction between targeting existing negative feelings and influencing the emotional environment in ways that may to lead to desired emotional outcomes. Although emotion regulation can be viewed as coping with an ongoing emotion, the process model of emotion regulation (Gross, 1998, 2015) emphasizes that emotions can also be influenced before the emotion even occurs. The model identifies situation selection (choosing or avoiding situations in ways that align with emotional goals) as the earliest opportunity for individuals to regulate the emotion generative cycle. Therefore, behavior aimed at avoiding or seeking out experiences based on how they may make you feel reflects emotion regulation. For instance, if you find the evening news upsetting, you may choose not to watch it.

Situation selection gives people an opportunity to avoid or limit engagement with negative emotional content, which may be useful for achieving high levels of emotional well-being (Charles, 2010). However, in typical laboratory paradigms participants are confronted with stimuli that they have little or no control over, and are instructed to regulate their emotions (e.g., "reduce negative affect", "suppress your emotional response so someone else would not be able to tell how you are feeling", or "look on the bright side"). These studies are important for informing us about the effectiveness of certain forms of emotion regulation but fail to capture the effect of potential age differences in the types of environments people select. Interestingly, despite reporting higher levels of emotional well-being in older age (e.g., Carstensen et al., 2000) older adults do not consistently outperform younger adults in traditional emotion regulation paradigms (see Lohani \& Isaacowitz, 2014; Opitz, Rauch, Terry, \& Urry, 2012; Shiota \& Levenson, 2009).

However, the ability to manage one's emotions during a standardized negative event does not seem to account for the age differences in everyday emotional experience (for a discussion of the distinction between emotion regulation behavior and downstream emotional consequences, see Isaacowitz \& Blanchard-Fields, 2012). This raises the question of whether other forms of emotion regulation are linked to better emotional outcomes in older age. Theoretically, situation selection has been proposed as an increasingly effective 
emotion regulation strategy in older age (Charles, 2010; Sands \& Isaacowitz, 2016; Urry \& Gross, 2010). Situation selection requires predicting emotional outcomes of various situations, but may be easier to implement than altering existing emotional responses as one ages (Charles, 2010). With age comes increased knowledge and experience with emotion regulation, which are theorized to lead to improvements in emotional domains (Charles, 2010).

\section{Selectivity in Older Age}

Selectivity is a key facet of several theories of healthy aging. Because of normative cognitive and physical declines, older adults need to select developmentally relevant and attainable goals to maintain general well-being and functioning (Baltes \& Baltes, 1990).

Socioemotional selectivity theory (SST; Carstensen, Isaacowitz, \& Charles, 1999) suggests that due to a more limited future time perspective, older adults value short-term emotional well-being over other longer-term goals (such as information acquisition).

One way to achieve these emotional well-being goals is to construct an environment that fosters positive emotional experiences and minimizes negative ones. As cognitive and physical resources decline with age, older adults may shift from relying on cognitively demanding strategies (e.g., reappraisal) to more antecedent-focused strategies that prevent emotions from occurring in the first place (e.g., situation selection; Urry \& Gross, 2010). For example, although older adults' social circles decrease in size with age, they are generally comprised of close and emotionally satisfying relationships (Carstensen, Fung, \& Charles, 2003). Older adults are also more likely to minimize social conflicts to preserve harmony within relationships (Birditt, Fingerman, \& Almeida, 2005). Thus, there is evidence, at least in the social domain, that older adults select some aspects of their environment in ways that maximize positive over negative emotions. One question is, does this selectivity generalize to non-social experiences as well? If so, what form would it take? For example, do older adults avoid negative and/or seek out positive experiences as emotion regulation to promote emotional well-being?

\section{An Age-Related Positivity Effect?}

Older adults preferentially process positive over negative emotional information-a phenomenon termed the age-related positivity effect. For younger adults, negative emotional information is typically the most salient, whereas older adults attend to and remember a greater ratio of positive compared to negative emotional information (Mather \& Carstensen, 2005; Reed, Chan, \& Mikels, 2014). For instance, older adults recall a higher ratio of positive to negative images than younger adults; younger adults are also better at identifying negative images, whereas older adults do not differ in recognition of positive and negative images (Charles, Mather, \& Carstensen, 2003). Increased attention to positive emotional stimuli is often interpreted as a means of supporting hedonic goals (Reed \& Carstensen, 2012). Preferential processing of positive emotional information has been linked to emotion regulation goals in some empirical studies (Isaacowitz, Toner, Goren, \& Wilson, 2008; Kennedy, Mather, \& Carstensen, 2004). 
Age-related positivity effects in cognitive processing have generally been studied in the context of controlled environments that present fixed stimuli to participants and measure participants' reactions. Although these paradigms allow us to examine complex cognitive and emotional processes, they may not fully reflect how emotion regulation works in younger and older adults' daily life, where they have a significant amount of control over the environments in which they operate. For several years, our lab has been examining how positivity effects may (or may not) occur in the ways that younger and older adults select the stimuli that they expose themselves to.

\section{Positivity Effects in Situation Selection?}

To address the question of whether people select more positive emotional environments as they get older, we developed an affective environment paradigm in which participants freely select from various affective stimuli for a period of time. In this paradigm, a "situation" is defined as an interaction with a particular stimulus, for example, viewing a particular video or reading an article. This paradigm allows us to mirror affect-laden choices that people make in their everyday lives-such as what shows to watch on television or what news articles to read - while evaluating their emotional experience in a controlled environment. Because participants have an array of affective selections from which to choose, we can assess the degree to which they choose pro-hedonically (i.e., more positive and less negative), in a way that might reflect greater motivation to experience positive and avoid negative emotions.

The first affective environment study did not find a main effect of age on the valence of selected situations, but found that older adults with higher emotional control beliefs (i.e., higher emotion regulation self-efficacy) were more likely to select positive emotional stimuli (Rovenpor, Skogsberg \& Isaacowitz, 2013). This suggested that at least a subset of older adults exhibited preferences for positive emotional situations. Subsequent studies assessed emotional outcomes and varied the stimuli and parameters to better understand the influence of arousal, goals, baseline mood, and other variables on age differences in selections (Isaacowitz, Livingstone, Harris, \& Marcotte, 2015; Isaacowitz, Livingstone, Richard, \& ElNasr, in prep; Livingstone \& Isaacowitz, 2015; Sands \& Isaacowitz, 2016; Sands, Garbacz \& Isaacowitz, 2016). Across studies, the situations people selected were closely related to their moods: For all ages, selecting positive situations was linked to more positive emotional outcomes than selecting negative situations. Interestingly, though, findings about age differences in the selections themselves were mixed. Some studies found that older adults expressed preferences for positivity or an avoidance of negative stimuli, whereas others did not.

\section{The Current Study}

Given the mixed findings across several studies with varying versions of the affective environment, it is important to determine whether there is an overall positivity effect in situation selection and, if so, which aspects of the situation moderate potential age differences. To test these questions, we conducted a "mini" meta-analysis on data collected in our lab. Mini meta-analysis is a recommended practice for summarizing a small group of 
conceptually similar studies, especially when there is not agreement in the conclusions drawn across studies (Goh, Hall \& Rosenthal, 2016). Additionally, this technique is useful because it allows researchers to include null findings while still interpreting the overall effect (thus reducing the file drawer problem), provides other researchers effect size information needed for power calculations, and can provide support for small or null effects that are difficult to establish in a single study (see Goh, Hall \& Rosenthal, 2016; Maner, 2014; Braver, Thoemmes \& Rosenthal, 2014). The statistical techniques are explained further below but are essentially the same as those used in traditional meta-analysis: An effect size is computed for each study and then an overall effect is determined using fixed or random effects models. Mini metas have been used to draw more robust conclusions in multi-study papers (e.g. Goh, Schlegel, Tignor \& Hall, 2016) and can be conducted with as few as two studies (e.g. Hugenberg \& Bodenhausen, 2004; Williams \& DeSteno, 2008). In the current study, we used these techniques to synthesize all the data collected in our lab from eight studies using the situation selection paradigm. ${ }^{1}$

In addition to overall positivity effects, our mini meta-analysis examined positivity approach vs. negativity avoidance, an important theoretical distinction in the literature. SST defines the age-related positivity effect as an age by valence interaction, resulting in older adults exhibiting a stronger preference for positive emotional content over negative emotional content compared to young adults. Since positivity effects are indicative of an age difference in how people engage with emotional content, SST theorizes that they can result from older adults' increased motivation to pursue pro-hedonic goals and/or young adults' increased motivation to pursue future-oriented goals, such as information seeking (Carstensen, Isaacowitz \& Charles, 1999). However, people can pursue pro-hedonic goals by seeking out positive emotional content or by avoiding negative, and SST does not clearly distinguish between positivity preference and negativity avoidance (Reed \& Carstensen, 2012; Reed et al., 2014). If positivity preference is the main mechanism, as SST suggests, we would expect older adults to prefer positive emotional content compared to neutral and negative information to a greater extent than younger adults. However, if negativity avoidance motivates positivity effects, we would expect older adults to prefer positive and neutral situations over negative to a greater extent than younger adults, but exhibit no differences in the positive vs. neutral comparison.

Given the variations in procedures across studies, we can also examine possible moderators that make positivity effects in selection more or less likely to emerge. Contextual factors, such as information presented about the choices (medium, content, valence category) or qualities of the stimuli could influence when age differences in selections emerge in individual studies. We analyzed three types of variables.

\footnotetext{
${ }^{1}$ The meta-analysis includes data from one unpublished study, which was designed to test the hypothesis that the salience of affective valence information could explain discrepant findings (i.e., if affective information about the selections is made more salient, an agerelated positivity effect may be more likely to emerge). 50 younger (ages 18-26) and 50 older (ages 60-89) adults completed a laboratory study in either the low-salience condition (valence of stimuli was not identified on computers or mentioned by the researcher) or a high-salience condition (valence of stimuli was clearly labeled on the computers and was mentioned by the researcher). Stimuli were those used in Isaacowitz et al. (2015). Participants made choices for 15 minutes and rated affect after each choice, as in previous studies. A 2 (age group) x 2 (valence salience condition) x 3 (selection valence) mixed ANOVA on the percentage of time spent engaging with information showed a main effect of valence, $F(2,93)=30.51, p<.001$ : Participants spent more time engaging with positive vs. neutral and negative stimuli. The main effects of age group and affective salience were not significant, $p s>.34$, nor were any interactions, $p s>.32$.
} 
First, variations in the qualities of the stimuli included as options in the study may have influenced decisions. For example, older adults may be particularly likely to avoid negative options when the options are particularly unpleasant or arousing, but may be less likely to avoid negative options when they are more relevant or informative. Emotionally-laden content can vary in informativeness; if younger adults are more motivated to engage with negative content because it is informative, age differences would be more likely in paradigms that included more informative stimuli. Recall studies have found that age differences are attenuated when negative content is more relevant or necessary for a task (Löckenhoff \& Carstensen, 2007). Therefore, more relevant stimuli may reduce age differences in situation selection behavior. On the other hand, arousal of the stimuli may also matter. Antecedent emotion regulation strategies like situation selection are theorized to be an effective way for older adults to avoid unpleasant increases in arousal, so studies with more activating stimuli might be associated with greater age differences in behavior.

Second, in some studies, participants were explicitly instructed to try to minimize their negative emotions. Introducing such a goal within a study may reduce age-related positivity effects, as both younger and older adults may use situation selection to regulate their emotions. Third, studies varied in the extent to which they emphasized the valence of the stimuli (emotional salience), which could potentially influence emotional goals. By analyzing across studies, we can synthesize the research about situation selection and better characterize the nuances of when positivity effects emerge in this paradigm (see Goh, Hall, $\&$ Rosenthal, 2016 for a review of mini-meta analysis).

\section{Method}

\section{Situation selection paradigm}

Our studies of situation selection have primarily utilized an affective environment paradigm in which participants spend 15 minutes freely selecting from emotional stimuli displayed on three different computer monitors and grouped by valence (i.e., positive, negative, and neutral; Figure 1). One study instead had individuals select stimuli from a single screen for 10 minutes with software that emulated television channels (Sands, Garbacz, \& Isaacowitz, 2016).

\section{Variations across studies}

These studies varied on a variety of parameters. Individual study details can be found in Table 1. Studies varied in a) stimulus characteristics, b) instructions to participants, and c) salience of the emotional content.

Stimuli characteristics-Specific stimuli options varied across studies. All stimuli were normed in samples of younger and older adults prior to inclusion in their respective studies; this normative data was used in the moderator analyses of stimuli characteristics, as study parameters might have influenced participants' ratings in the affective environment paradigm. Valence (positivity/negativity), arousal/activation, and relevance ${ }^{2}$ were rated on

\footnotetext{
${ }^{2}$ Relevance ratings were not available for Sands, Garbacz, \& Isaacowitz (2016).
} 
continuous scales; these were transformed to POMP scores (Cohen, Cohen, \& Aiken, 1999) to compare across studies. Stimuli were originally selected for use because they did not have age differences on these dimensions. We therefore computed scores across age groups for all stimuli in the study (overall scores) as well as for each valence separately.

Because the studies were more concerned with equating affective qualities of the stimuli across age groups, informational value was not originally assessed or manipulated. However, as informational goals are one explanation for why younger adults may engage with negative content (Carstensen et al., 1999), we assessed the average informational value of the stimuli at the study level to determine if studies with greater informational value were more likely to show age differences. For the purpose of the meta-analysis, two trained coders, blind to the purpose of the analyses, identified the percentage of stimuli in each study that were "informative," or that specifically taught participants new information (informative items included instructional videos, historical accounts, and health-related news segments; noninformative items included commercial movie clips, home videos, and human interest stories). Disagreements were resolved through discussion moderated by the second author.

Instructions to participants-In some studies, groups of participants were instructed to "minimize [their] negative feelings" during their selection period. In these studies, a control group received instructions to "choose what is interesting to you."

Salience of emotional content-In some studies, participants were explicitly told that they could select from positive, negative, and neutral stimuli and were informed of the location of each of these types of content (high salience). In other studies, participants were just told they could select whatever they wanted, for however long they wanted, without mention of the emotional nature of the videos (low salience).

\section{Statistical analyses}

We conducted three separate mini meta-analyses (see Goh, Hall \& Rosenthal, 2016) to compare selections between each pair of valence categories: neutral vs. negative, negative vs. positive, positive vs. neutral. Within studies, we conducted a 2 (age group) x 2 (valence) mixed model analysis of variance (ANOVA) for each comparison. We used the age by valence interaction term to compute an effect size $(r)$ for each comparison, resulting in three effect sizes for each study. Each of these effect sizes were entered into separate metaanalyses each comparing two valence categories, which allowed us to examine all the theoretically relevant comparisons. This resulted in one data point from each study for each meta-analysis. All correlations were normalized with the Fisher's $z$ transformation prior to being used for statistical calculations. For clarity in interpretation, they are presented as Pearson correlations in this manuscript. A positive sign (+) for effect sizes indicated that older adults exhibited a stronger bias towards positive information and/or younger adults exhibited a stronger bias towards negative information.

Meta-analyses can use either fixed or random effects models. Fixed effects models assume there is one true effect size across studies and weight effect size by sample size (e.g. larger samples are given more weight when calculating the mean effect size across studies). Random effects models, on the other hand, assume that there is variance in the true effect 
size across studies due to differences in methodology and/or sampling. We used random effects models for all analyses because our studies had key differences in design and we wanted to maximize generalizability (see; Borenstein, Hedges, Higgins, \& Rothstein, 2009; Lipsey \& Wilson, 2001). Analyses were conducted using Comprehensive Meta Analysis software (CMA; Biostat, 2014).

Moderator analyses-We also used CMA to conduct a series of moderator analyses to examine how contextual differences across paradigms related to study effect size. These analyses allowed us to statistically test whether theoretically relevant study level variables including characteristics of the stimuli (i.e., arousal level, informativeness, negativity) and aspects of the paradigm (i.e., emotion regulation instructions, valence information salience) influenced differences in effect sizes among studies. For categorical moderators, we used Hedges' Q-between for random effects which compares the heterogeneity in effect sizes within a category with the heterogeneity across categories (similar to ANOVA; Borenstein et al., 2009). The mean effect size is computed for each group (e.g., emotion regulation instructions vs. no instructions) and statistically compared to determine if the groups of studies differ in effect size. For instance, is the effect size in studies with emotion regulation instructions different from studies that did not instruct participants to regulate their emotions? For continuous variables, we used meta- regression, which, rather than testing group differences, determines whether there is a relationship between continuous moderator variables and effect sizes (Borenstein et al., 2009; Hedges \& Olkin, 1985). For example, are studies with higher-arousal stimuli associated with larger effect sizes? When interpreting these analyses, it is important to remember that the moderator is coded at the study level (and is not an analysis of the variance within studies).

\section{Results}

\section{Age-related Positivity Effects}

We conducted three analyses to examine age-related positivity effects as traditionally defined (positive vs. negative; Reed et al., 2014), along with tests of positivity preference and negativity avoidance.

Positive vs. negative selections-Across 14 samples, there was no significant age by valence interaction when comparing positive and negative selections, $M_{r}=0.076,95 \% \mathrm{CI}$ [-.013, 0.163], $Z=1.67, p=0.095$. Thus, the overall positivity effect, as traditionally defined, did not emerge (see Table 2).

Positive vs. neutral selections-We conducted a separate meta-analysis to examine if there were age differences in selections of positive compared to neutral situations (positivity preference). Across 14 samples, the age by valence interaction was not significant, $M_{r}=$ $0.024,95 \% \mathrm{CI}[-0.056,0.104], Z=.587, p=0.557$ suggesting that there was not an increased preference for positive situations with age (see Table 3).

Negative vs. neutral selections-Finally, we conducted a third meta-analysis to examine if there were age differences in negative compared to neutral selections (negativity avoidance). The age by valence interaction was not significant, $M_{r}=0.181,95 \%$ CI [ -0.045 , 
$0.389], Z=1.57, p=0.116$, indicating that there were not age differences in negativity avoidance (see Table 4).

\section{Moderator Analyses}

We conducted moderator analyses to examine the influence of contextual differences in the paradigm on age differences in selections. These analyses examine whether features of the individual studies predict variation in effect size. All moderator analyses were conducted separately for each meta-analysis.

Stimuli characteristics-For the positive vs. negative comparison, overall relevance ( $p=$ $0.265)$, informativeness ( $p=.254)$, arousal $(p=0.49)$, and negativity $(p=.75)$ did not moderate effect size. For the positive vs. neutral comparison, negativity $(p=.568)$, informativeness $(p=0.536)$, arousal $(p=.17)$, and relevance $(p=.18)$ did not moderate effect size.

For the negative vs. neutral comparison, the effect size was larger when overall stimulus relevance $(p=0.010)$ and the relevance of the negative content specifically $(p<.001)$ were higher, indicating that in studies in which stimuli were more relevant, younger adults were less likely to avoid negative content than older adults when stimuli are more relevant. Additionally, the arousal of the stimuli moderated effect size; higher arousal positive stimuli ( $p=0.033$ ) were associated with studies with larger effect sizes. In other words, when positive stimuli were more arousing, older adults were more likely to choose neutral over negative stimuli. However, average stimulus negativity $(p=.120)$, the arousal level of negative stimuli $(p=0.099)$ and informativeness $(p=0.374)$ were not significant moderators of age effects.

Emotion regulation instructions-For the positive vs. negative comparison, studies with emotion regulation instructions $(M r=.087)$ and studies without instructions $(M r=$. 036) did not differ in effect size $(p=.635)$. For the positive vs. neutral comparison, studies without regulation instructions $(M r=.003)$ did not differ in effect size from studies with regulation instructions $(M r=.087 ; p=.434)$. For the negative vs. neutral comparison, studies without regulation instructions $(M r=0.238)$ did not have significantly larger effect sizes than studies with regulation instructions $(M r=-0.028 ; p=.320)$.

Salience of Emotional Valence-For the positive vs. negative comparison, studies in which instructions made the emotional valence of the stimuli more salient $(M r=0.136)$ did not significantly differ from studies that did not $(M r=-0.007 ; p=0.08)$. For the positive vs. neutral comparison, studies with high salience $(M r=-0.005)$ did not differ from studies with low salience ( $M r=0.070, p=.421)$. For the negative vs. neutral comparison, studies with high salience $(M r=.265)$ did not differ from studies with low salience $(M r=0.031, p$ $=.304)$.

\section{Discussion}

In sum, these findings do not support the idea of an overall age difference in situation selection, at least using the affective environment paradigm. In the three meta-analyses we 
conducted, there were no consistent valence by age interactions in selection behavior. Older adults did not show a significant increase in preferences for positive stimuli or an increase in avoidance of negative stimuli across studies, compared to younger adults, suggesting that there is not a global age-related positivity effect in situation selection. However, moderator analyses and individual studies do give us a more nuanced perspective about the contexts in which age differences are more likely to occur in selection behavior. Of importance were relevance and arousal ratings of the stimuli in the negative vs. neutral comparison.

For the meta-analytic comparison of negative vs. neutral selections, age-related positivity effects were more likely to occur when the stimuli, particularly negative stimuli, were rated as more relevant. It is important to note that older adults are not avoiding negative stimuli overall; they are avoiding more relevant negative stimuli compared to younger adults.

Additionally, for the negative vs. neutral comparison, the arousal level of the stimuli moderated age differences. If the positive stimuli were more arousing, age differences in selections were greater (i.e., older adults were more likely to select neutral over negative).

\section{Implications for the Study of Age-Related Positivity Effects}

A strong SST perspective would predict that older adults preferentially process positive content to serve emotion regulatory goals that become more important as time becomes more limited. This has generally been the case of cognitive processing in laboratory studies in which the stimuli are controlled by the researcher (Reed et al., 2014). The findings reported here do not support this strong claim when stimuli are freely chosen. In contrast to laboratory studies on attention and memory in which older adults, to a greater extent than young adults, preferentially process positive over negative material (Reed et al., 2014), situation selection does not show this pattern. Overall, there is no evidence for a global positivity preference in selections. However, findings were nonsignificant but numerically in the predicted direction for the negative vs. neutral and negative vs. positive comparisons. Additionally, significant moderators emerged for the negative vs. neutral comparison. Though a strong SST perspective is not supported, this mini meta-analysis does suggest that older adults are more likely than younger adults to show negativity avoidance in some contexts, such as when the available options are more relevant and arousing.

Motivation in situation selection-According to SST, older adults' emotional wellbeing goals are more chronically active, whereas younger adults' goals are more situationally variable. In younger adulthood, when time horizons are expansive, younger adults can face considerable long-term gain by gathering information and experiences, though they may be unpleasant. Younger adults may be more likely, therefore, to engage with negative material, whereas when content is less relevant or informative, emotional wellbeing goals may take priority.

Though age differences were stronger with greater relevance, the effects were not large, and positivity preference seemed to be the driving motivation for both younger and older adults. It may be the case that positivity effects in cognitive processing in older age occur mainly in reaction to uncontrollable stimuli, which may be rare in everyday life. This is in line with 
research showing that positivity effects in attention do not appear when stimuli are selfselected (Isaacowitz et al., 2015).

It is unclear whether these few age differences, when they do emerge, are accounted for by older adults' increased pro-hedonic motivation, young adults' future oriented goals, or both. Interestingly, informativeness was not a significant moderator of age differences, indicating that in this context younger adults do not seem to be prioritizing information seeking goals more than older adults. The affective environment does not directly assess motivation; rather, we infer motivation based on selections. It may therefore be useful to directly assess participants' motivations while engaging in the affective environment. For example, one previous study showed that age differences disappeared when both younger and older adults were assigned emotion regulation goals (Livingstone \& Isaacowitz, 2016). However, because informational value was not manipulated, the affective environment studies described here are best suited towards evaluating emotional goals. Future studies could also systematically manipulate informational value or assign information-seeking goals to better understand the interplay between emotion regulation and information-seeking goals when selecting situations.

Other research has shown that older adults are more likely to remember negative information when it is relevant to a future decision (Depping \& Freund, 2013). Age differences in selecting information also disappear when participants are given an information-gathering goal when reviewing health-related information (Löckenhoff \& Carstensen, 2007). In these studies, when negative information was known to be immediately useful (e.g., for future recall), positivity effects disappeared or were reversed. In contrast, in the current study, any information acquired would only be useful in the more distal future (e.g., learning about malaria because you want to go to Africa). Moreover, although our task shares some overlap with general information-seeking tasks, the fact that choices are valenced in nature makes the affective environment more suitable for studying emotion regulation-relevant behavior rather than general curiosity.

Though relevance, informativeness, and utility are all operationalized differently in these studies, there is conceptual overlap between these constructs. For instance, emotional content could be personally relevant to someone without providing new or useful information (e.g., related to a past experience), but at other times, content may only be relevant because of its utility. Future research could make a significant theoretical contribution by disentangling these constructs to determine how they are related to contrahedonic motivations. Additionally, it would be useful to examine situations in which younger adults may pursue contra-hedonic goals for emotional reasons (e.g., Tamir, 2009).

Arousal-focused theories-Older adults were more likely to select neutral than negative stimuli, compared to young adults, if the positive stimuli were more arousing. Since neutral stimuli are generally low in arousal, this is consistent with the finding that older adults are more likely to prefer low-arousal content, regardless of emotional valence (Sands \& Isaacowitz, 2016). This finding aligns well with the theoretical perspective that older adults may have more difficulty regulating physiological responses to highly arousing material, due to biological changes in the autonomic nervous system (Charles, 2010; Shiota \& Neufeld, 
2014). As people age, physiological reactivity is prolonged and may become increasingly unpleasant, which may motivate older adults to avoid activating contexts regardless of valence (Charles, 2010; Sands, Garbacz \& Isaacowitz, 2016).

\section{Implications for the Study of Emotion Regulation}

According to the process model of emotion regulation, intervening early in the emotiongenerative process is often more effective than trying to intervene later, when the emotional experience is already underway (Gross, 1998). This is thought to be especially important for emotion regulation in older age (Charles, 2010; Urry \& Gross, 2010). Thus, in addition to investigating positivity effects in cognitive processing of already existing emotional situations, it is critical to understand positivity effects in all stages of the emotion regulation process, including situation selection. Rather than revealing a consistent age difference in preferences for emotional situations, however, our findings show that younger and older adults only differ in their selections in some contexts.

Although not tested here, all the studies summarized in this manuscript suggest that positivity preference and negativity avoidance in situation selection are clearly linked with more positive emotional experience. This does not appear to vary by age, indicating that the effectiveness of situation selection is consistent across age groups (see Livingstone \& Isaacowitz, 2015; Sands \& Isaacowitz, 2016). The research presented here does not support the claims that positivity in situation selection is more frequently used or that it is more effective in older age. Instead, situation selection may be important because its use and effectiveness are consistent across the lifespan, whereas other strategies may become less effective with age (e.g., detached reappraisal; Shiota \& Levenson, 2009). It will also be important to link choices of situations in everyday life to long term emotional well-being.

\section{Limitations}

The extent to which people utilize situation selection in real life is yet unclear, but people typically have more control over their environments in real life than they do in the lab. Furthering our understanding of positivity effects in emotion regulation, therefore, will require both laboratory studies with high internal validity and more ecologically valid assessments such as experience sampling. Such research may identify ways in which younger and older adults avoid and enter situations in their everyday life; this may reveal patterns that differ from laboratory paradigms. On the other hand, situation selection is also likely to be more complex in real life. For example, the affective environment studies presented here did not allow opportunity for social interaction, or time to work toward a personal goal. When such options are present, positivity effects in situation selection may be more likely to emerge. Similarly, if laboratory stimuli were even more personally relevant, informative, or useful for a subsequent task, younger adults may be more motivated to engage with the negative material compared to older adults.

Although the moderator analyses suggest that some contextual factors that may make negativity avoidance in situation selection more or less likely, we could not test all possible moderators due to insufficient variance. In addition, these results should be interpreted with some caution since the number of studies is somewhat small and each of these studies was 
designed to test specific questions (e.g., regarding the role of goals, arousal information, etc.). Our aim here was to summarize our knowledge of situation selection behavior using the affective environment to date. As Webb and colleagues noted in their meta-analysis, research on situation selection is far rarer than cognitive and response-based emotion regulation strategies (Webb, Miles, \& Sheeran, 2012). Future research and other labs should therefore extend beyond this paradigm to test additional hypotheses about when positivity effects may or may not emerge (for research on situation selection in younger adults, see Markovitch, Netzer, \& Tamir, in press; Vujovic, Opitz, Birk, \& Urry, 2014).

\section{Conclusion}

Older adults generally report high levels of emotional well-being, and situation selection is theorized to be a key strategy by which they achieve this. However, we did not find evidence that older adults are more likely than younger adults overall to use situation selection to seek out more positive emotional situations. Both younger and older adults prefer positive emotional situations unless other contextual factors, such as relevance or arousal, motivate individuals to select other types of emotional stimuli. In these contexts, age differences in preferences did vary in the predicted direction, with older individuals avoiding negative information to a greater extent than younger adults. Although we did not find strong support for age-related differences in situation selection, we maintain that situation selection may nonetheless be an important strategy in older age, as in younger ages as well.

\section{Acknowledgments}

This research was supported by NIA grants R21AG044961 and R01AG048731 to the last author.

\section{References}

Baltes PB, Baltes MM. Psychological perspectives on successful aging: The model of selective optimization with compensation. In: Baltes PB, Baltes MM, editorsSuccessful aging: Perspectives from the behavioral sciences. New York: Cambridge University Press; 1990. 1-34.

Birditt KS, Fingerman KL, Almeida DM. Age differences in exposure and reactions to interpersonal tensions: A daily diary study. Psychology and Aging. 2005; 20:330-340. DOI: 10.1037/0882-7974.20.2.330 [PubMed: 16029096]

Braver SL, Thoemmes FJ, Rosenthal R. Continuously cumulating meta-analysis and replicability. Perspectives on Psychological Science. 2014; 9(3):333-342. [PubMed: 26173268]

Borenstein M, Hedges LV, Higgins JPT, Rothstein HR. Introduction to meta-analysis. Chichester, UK: Wiley; 2009.

Carstensen LL, Fung HH, Charles ST. Socioemotional selectivity theory and the regulation of emotion in the second half of life. Motivation and Emotion. 2003; 27:103-123. DOI: 10.1023/A: 1024569803230

Carstensen LL, Isaacowitz DM, Charles ST. Taking time seriously: A theory of socioemotional selectivity. American Psychologist. 1999; 54:165-181. DOI: 10.1037/0003-066x.54.3.165 [PubMed: 10199217]

Charles ST. Strength and vulnerability integration: A model of emotional well-being across adulthood. Psychological Bulletin. 2010; 136:1068-1091. DOI: 10.1037/a0021232 [PubMed: 21038939]

Charles S, Mather M, Carstensen LL. Aging and emotional memory: The forgettable nature of negative images for older adults. Journal of Experimental Psychology: General. 2003; 132:310-324. DOI: 10.1037/0096-3445.132.2.310 [PubMed: 12825643]

Cohen P, Cohen J, Aiken LS, West SG. The problem of units and the circumstance for POMP. Multivariate behavioral research. 1999; 34:315-346. DOI: 10.1207/s15327906mbr3403_2 
Depping MK, Freund AM. When choice matters: Task-dependent memory effects in older adulthood. Psychology and Aging. 2013; 28:923.doi: 10.1037/a0034520 [PubMed: 24364399]

Goh JX, Hall JA, Rosenthal R. Mini meta-analysis of your own studies: Some arguments on why and a primer on how. Social and Personality Compass. 2016; 10:535-549. DOI: 10.1111/spc3.12267

Goh JX, Schlegel K, Tignor SM, Hall JA. Who is interested in personality? The Interest in Personality Scale and its correlates. Personality and Individual Differences. 2016; 101:185-191.

Gross JJ. The emerging field of emotion regulation: An integrative review. Review of General Psychology. 1998; 2:271-299. DOI: 10.1037/1089-2680.2.3.271

Gross JJ, Thompson RA. Emotion regulation: Conceptual foundations. In: Gross JJ, Thompson RA, editorsHandbook of emotion regulation. Guilford Press; New York: 2007. 3-24.

Hedges L, Olkin I. Statistical methods for meta-analysis. New York: Academic Press; 1985.

Hugenberg K, Bodenhausen GV. Ambiguity in social categorization: The role of prejudice and facial affect in race categorization. Psychological Science. 2004; 15(5):342-345. [PubMed: 15102145]

Isaacowitz DM, Livingstone KM, Harris JA, Marcotte SL. Mobile eye tracking reveals little evidence for age differences in attentional selection for mood regulation. Emotion. 2015; 15:151-161. DOI: 10.1037/emo0000037 [PubMed: 25527965]

Isaacowitz DM, Livingstone KM, Richard M, El-Nasr M. Aging and attention to self-selected emotional content: A novel application of mobile eye tracking to the study of emotion regulation. in prep.

Isaacowitz DM, Toner K, Goren D, Wilson HR. Looking while unhappy: Mood-congruent gaze in young adults, positive gaze in older adults. Psychological Science. 2008; 19:848-853. DOI: 10.1037/a0026666 [PubMed: 18947348]

Kennedy Q, Mather M, Carstensen LL. The role of motivation in the age-related positivity effect in autobiographical memory. Psychological Science. 2004; 15:208-214. DOI: 10.1111/j. 0956-7976.2004.01503011.x [PubMed: 15016294]

Livingstone KM, Isaacowitz DM. Situation selection and modification for emotion regulation in younger and older adults. Social Psychological and Personality Science. 2015; 6:904-910. DOI: 10.1177/1948550615593148 [PubMed: 26998196]

Maner JK. Let's put our money where our mouth is if authors are to change their ways, reviewers (and editors) must change with them. Perspectives on Psychological Science. 2014; 9(3):343-351. [PubMed: 26173269]

Mather M, Carstensen LL. Aging and motivated cognition: The positivity effect in attention and memory. Trends in Cognitive Sciences. 2005; 9:496-502. DOI: 10.1016/j.tics.2005.08.005 [PubMed: 16154382]

Lipsey MW, Wilson DB. Practical meta-analysis. Thousand Oaks, CA: Sage; 2001.

Löckenhoff CE, Carstensen LL. Aging, emotion, and health-related decision strategies: motivational manipulations can reduce age differences. Psychology and Aging. 2007; 22:134-146. DOI: 10.1037/0882-7974.22.1.134 [PubMed: 17385990]

Lohani M, Isaacowitz DM. Age differences in managing response to sadness elicitors using attentional deployment, positive reappraisal, and suppression. Cognition and Emotion. 2014; 28:678-697. DOI: 10.1080/02699931.2013.853648 [PubMed: 24206128]

Markovitch N, Netzer L, Tamir M. What you like is what you try to get: Attitudes toward emotions and situation selection. Emotion. in press.

Opitz PC, Rauch LC, Terry DP, Urry HL. Prefrontal mediation of age differences in cognitive reappraisal. Neurobiology of Aging. 2012; 33:645-655. DOI: 10.1016/j.neurobiolaging. 2010.06.004 [PubMed: 20674090]

Reed AE, Carstensen LL. The theory behind the age-related positivity effect. Frontiers in Emotion Science. 2012; 3:1-9. DOI: 10.3389/fpsyg.2012.00339

Reed AE, Chan L, Mikels JA. Meta-analysis of the age-related positivity effect: Age differences in preferences for positive over negative information. Psychology and Aging. 2014; 29:1-15. DOI: 10.1037/a0035194 [PubMed: 24660792]

Rovenpor D, Skogsberg N, Isaacowitz DM. The choices we make: An examination of situation selection in younger and older adults. Psychology and Aging. 2013; 28:365-376. DOI: 10.1037/ a0030450 [PubMed: 23088197] 
Sands M, Garbacz A, Isaacowitz DM. Just change the channel? Studying effects of age on emotion regulation using a TV watching paradigm. Social Psychological and Personality Science. 2016; :18. DOI: $10.1177 / 1948550616660593$

Sands M, Isaacowitz DM. Situation selection across adulthood: The role of arousal. Cognition and Emotion. 2016; :1-8. DOI: 10.1080/02699931.2016.1152954

Scheibe S, Mata R, Carstensen LL. Age differences in affective forecasting and experienced emotion surrounding the 2008 US presidential election. Cognition \& Emotion. 2011; 25:1029-1044. DOI: 10.1080/02699931.2010.545543 [PubMed: 21547760]

Shiota MN, Levenson RW. Effects of aging on experimentally instructed detached reappraisal, positive reappraisal, and emotional behavior suppression. Psychology and Aging. 2009; 24:890-900. DOI: 10.1037/a0017896 [PubMed: 20025404]

Shiota MN, Neufeld SL. My heart will go on: Aging and autonomic nervous system responding in emotion. In: Verhaegen P, Herzog C, editorsThe Oxford handbook of emotion, social cognition, and problem solving in adulthood. New York: Oxford University Press; 2014. 225-235.

Tamir M. What do people want to feel and why? Pleasure and utility in emotion regulation. Current Directions in Psychological Science. 2009; 18:101-105. DOI: 10.1111/j.1467-8721.2009.01617.x

Urry HL, Gross JJ. Emotion regulation in older age. Current Directions in Psychological Science. 2010; 19:352-357. DOI: 10.1037/14857-004

Vujovic L, Opitz PC, Birk JL, Urry HL. Cut! that's a wrap: Regulating negative emotion by ending emotion-eliciting situations. Frontiers in Psychology. 2014; 5doi: 10.3389/fpsyg.2014.00165

Webb TL, Miles E, Sheeran P. Dealing with feeling: a meta-analysis of the effectiveness of strategies derived from the process model of emotion regulation. Psychological bulletin. 2012; 138(4):775808. DOI: 10.1037/a0027600 [PubMed: 22582737]

Williams LA, DeSteno D. Pride and perseverance: the motivational role of pride. Journal of personality and social psychology. 2008; 94(6):1007-1017. [PubMed: 18505314] 


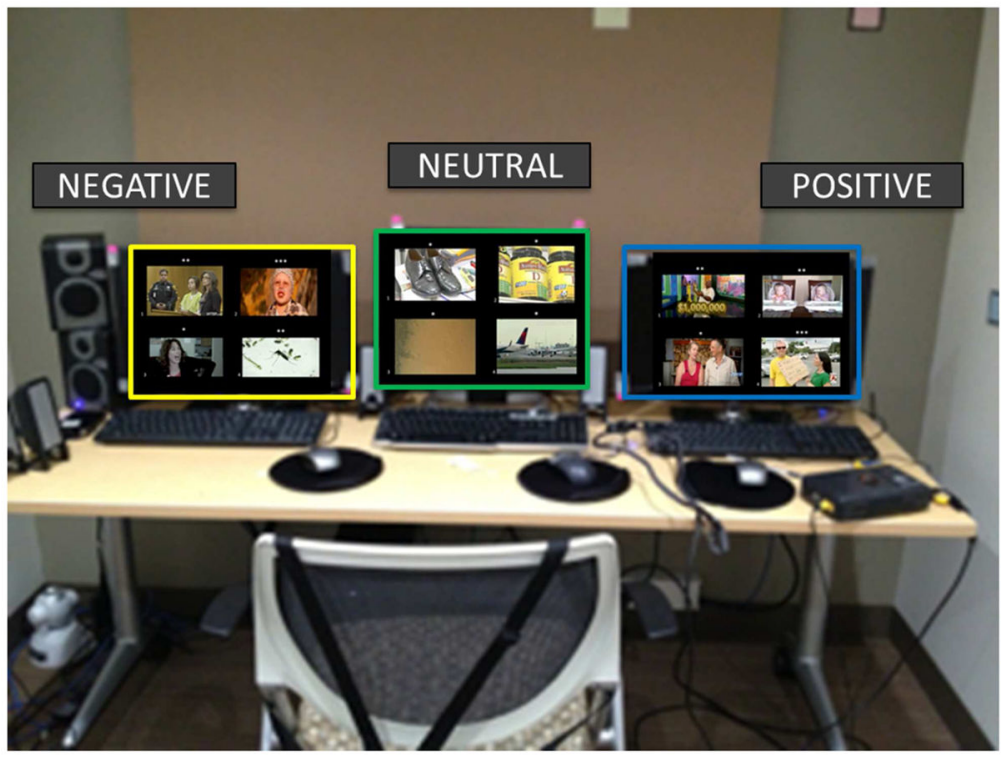

Figure 1.

Affective environment set up with computer monitors displaying positive, negative and neutral information. 


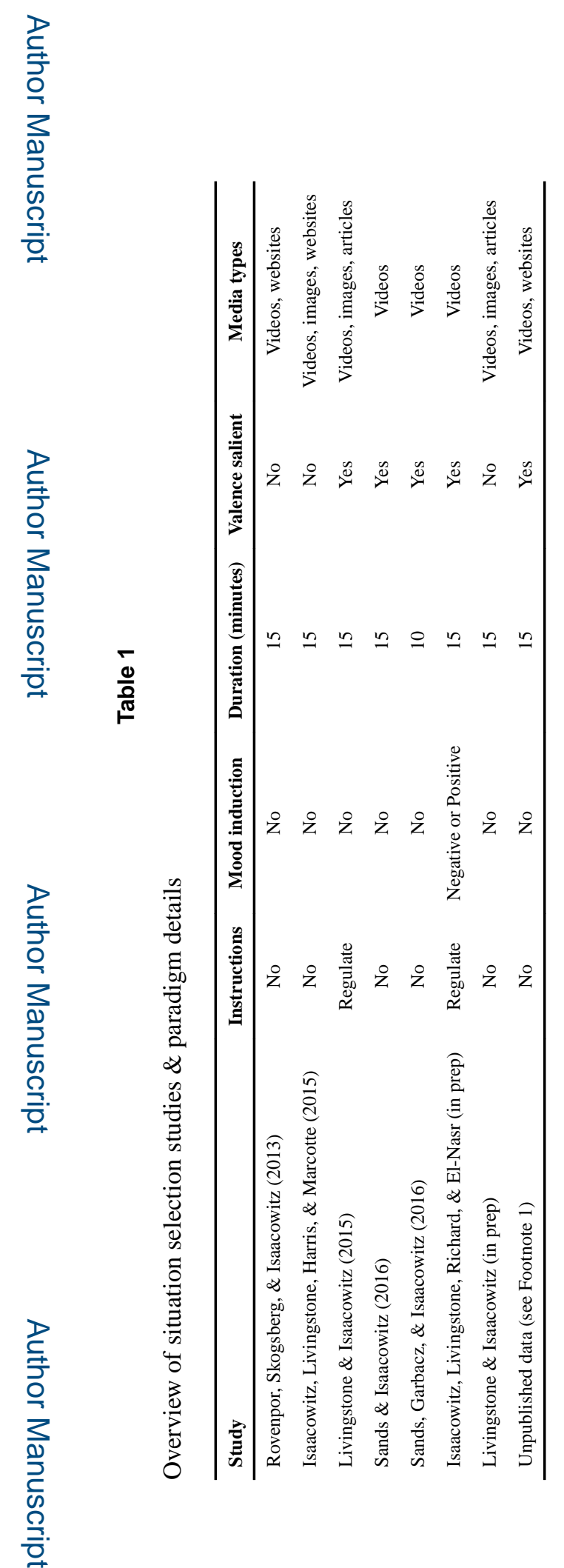

Int J Behav Dev. Author manuscript; available in PMC 2019 July 01. 


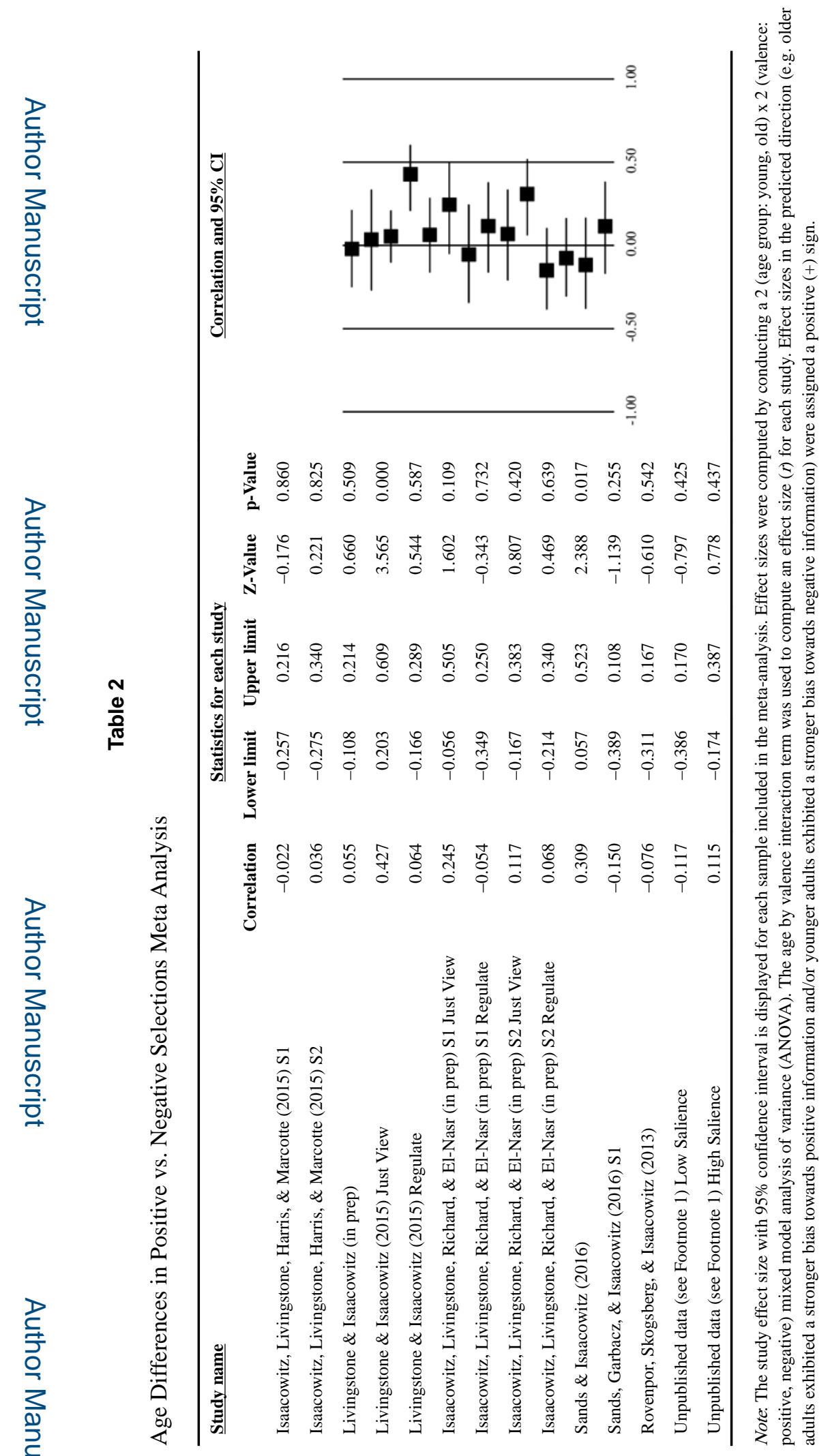

Int J Behav Dev. Author manuscript; available in PMC 2019 July 01. 


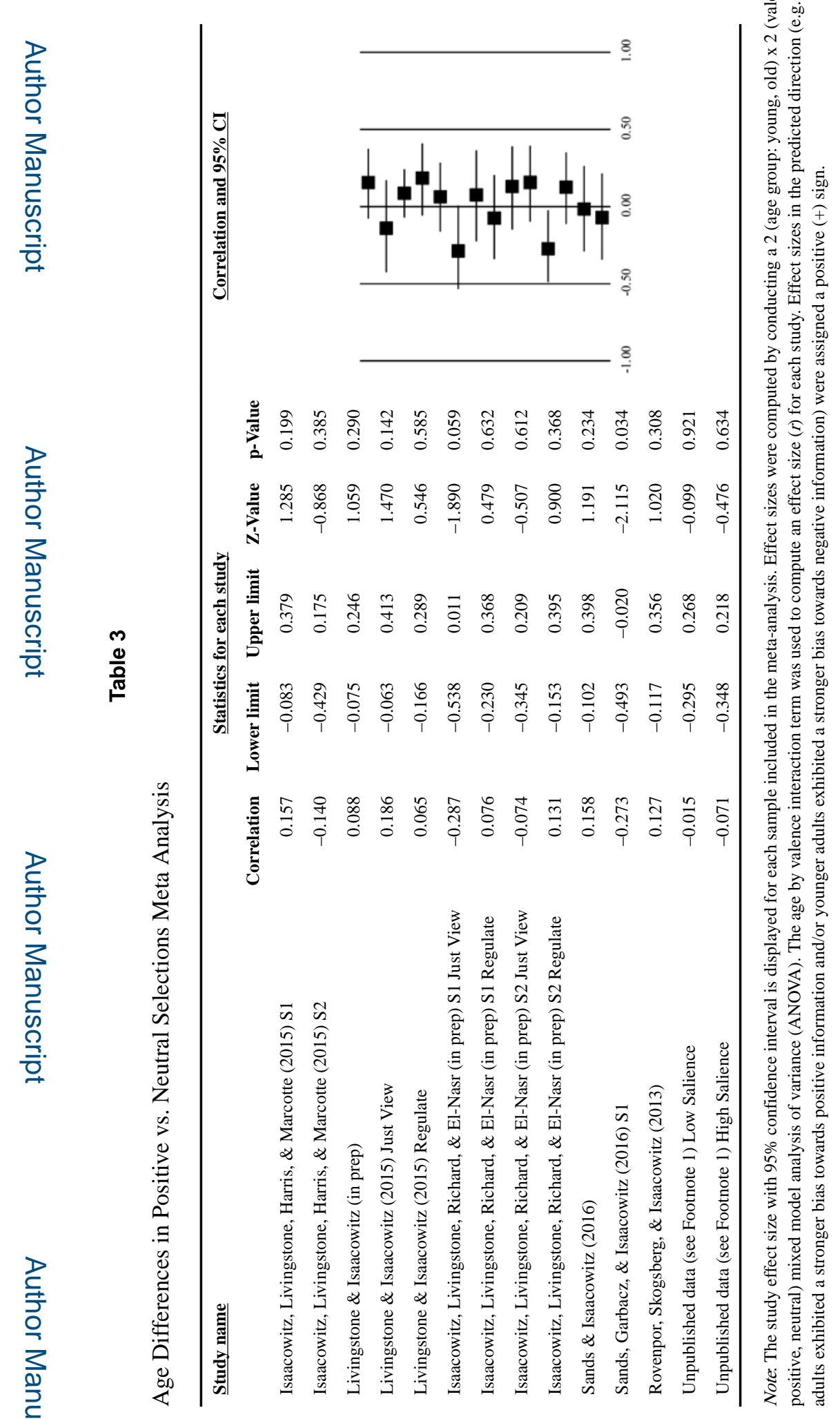

Int J Behav Dev. Author manuscript; available in PMC 2019 July 01. 

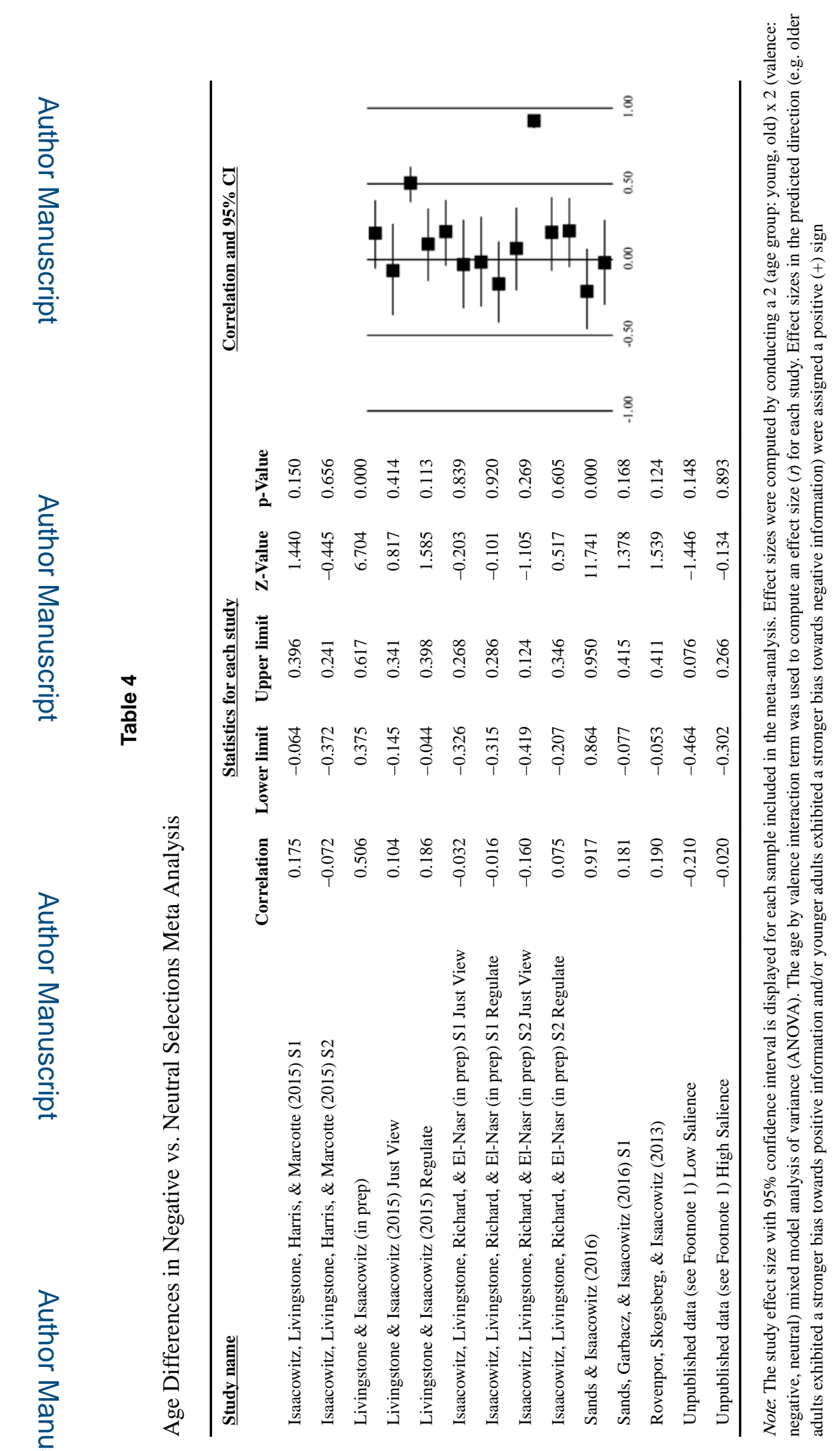

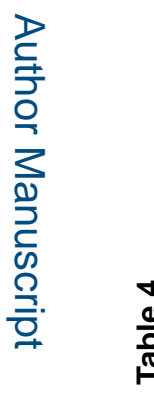

ב

Int J Behav Dev. Author manuscript; available in PMC 2019 July 01. 